\title{
A Comparative Study of the Effect of Gold Seed Particle Preparation Method on Nanowire Growth
}

\author{
Maria E. Messing ${ }^{1}(\bowtie)$, Karla Hillerich ${ }^{1}$, Jessica Bolinsson ${ }^{1}$, Kristian Storm ${ }^{1}$, Jonas Johansson ${ }^{1}$, Kimberly A. Dick ${ }^{1,2}$, \\ and Knut Deppert ${ }^{1}$ \\ ${ }^{1}$ Solid State Physics, Lund University, Box 118, S-221 00 Lund, Sweden \\ ${ }^{2}$ Polymer \& Materials Chemistry, Lund University, Box 124, S-221 00 Lund, Sweden \\ Received: 19 April 2010 / Revised: 19 May 2010 / Accepted: 19 May 2010 \\ C The Author(s) 2010. This article is published with open access at Springerlink.com
}

\begin{abstract}
Highly controlled particle-assisted growth of semiconductor nanowires has been performed for many years, and a number of novel nanowire-based devices have been demonstrated. Full control of the epitaxial growth is required to optimize the performance of devices, and gold seed particles are known to provide the most controlled growth. Successful nanowire growth from gold particles generated and deposited by various different methods has been reported, but no investigation has yet been performed to compare the effects of gold particle generation and deposition methods on nanowire growth. In this article we present a direct comparative study of the effect of the gold particle creation and deposition methods on nanowire growth characteristics and nanowire crystal structure, and investigate the limitations of the different generation and deposition methods used.
\end{abstract}

\section{KEYWORDS}

Nanowire growth, nanoparticles, gold, epitaxy, electron microscopy

\section{Introduction}

Semiconductor nanowires attract considerable attention for their potential as building blocks for the creation of novel electronic, optoelectronic and thermoelectronic devices [1]. Nanoparticles of various different materials have been used to seed the growth of nanowires, but gold particles are by far the most common choice [2]. Successful and highly controlled nanowire growth has been reported using gold particles generated and deposited by various methods, including aerosol-generated gold particles [3], colloidal gold particles [4], gold particles generated by annealing of thin films [5] and electron beam lithography- (EBL-) defined gold particles [6]. A few reports of nanowire

Address correspondence to maria.messing@ftf.lth.se growth from gold particles defined by other methods such as nanoimprint lithography [7] and laser ablation [8] can also be found, but such techniques are less common and therefore will not be considered in this article. Gold particles generated and deposited in different ways exhibit differences in controllability of size, position, surface density, and cleanliness. However, no direct comparison of the effect of gold particle preparation method on nanowire growth has been reported. Therefore it is still unclear whether nanowire growth studies performed in similar systems can be directly compared, when the gold particles used to seed the growth have been prepared by different methods.

We have investigated the effect of gold particle preparation method on the growth of GaAs nanowires 
by metal organic vapor phase epitaxy (MOVPE). We use "particle type" to indicate gold particles generated and deposited with a specific method. Gold particles generated by annealing of thin films (TFA), colloidal gold particles deposited by direct deposition (DDC) and by electrospray deposition (ESC), aerosol gold particles generated by evaporation/condensation (ECA) and by spark discharge (SDA) and EBL-defined gold particles (EBD) were used as nanowire seed particles, and the resulting nanowires (Fig. 1) were directly compared. These acronyms for the different particle types will be used throughout the paper to facilitate further reading. General differences in the seed particle characteristics induced by the particle preparation method as well as comparisons of the incubation time, growth related characteristics and crystal structure between nanowires seeded with the different particle types are reported.

\section{Experimental}

All particle types were prepared on GaAs (111)B oriented substrates. Two different kinds of samples were prepared for the different particle types with particle diameters of 30 and $80 \mathrm{~nm}$, respectively, both having a particle surface density of 1 particle/ $\mu \mathrm{m}^{2}$. This was not possible for all preparation methods and will be discussed in detail in Section 3.1 below.

Particles generated from thin films (TFA) were prepared by thermal evaporation (AVAC) of a thin gold film directly onto the substrate. The $1 \mathrm{~nm}$ thick film was deposited with a deposition rate of $0.1 \mathrm{~nm} / \mathrm{s}$, measured with a quartz crystal monitor. Following deposition of the thin film, the substrates were transferred in air to the reactor cell in the MOVPE system and during an in situ annealing step at $650{ }^{\circ} \mathrm{C}$ for $6 \mathrm{~min}$ prior to growth the thin film split up into particles.

Colloid particles from commercially available colloidal solutions (British Biocell International) were deposited by two different methods: the direct deposition method and the electrospray method [9]. For the direct deposition method, the substrates were first covered with a $0.1 \%$ poly- $L$-lysine (PLL) solution for $1 \mathrm{~min}$ and rinsed with deionized water (DIW) in order to ensure adhesion of the particles to the substrate by electrostatic interactions [4]. A droplet of colloidal solution with a concentration of $1.8 \times 10^{12}$ and $1.9 \times 10^{11}$ particles $/ \mathrm{cm}^{3}$ for particle diameters of 30 and $80 \mathrm{~nm}$ respectively was spin-coated onto the substrate in order to obtain an even surface distribution and to remove excess liquid. In the $30 \mathrm{~nm}$ case the solution rested for $30 \mathrm{~s}$ and was subsequently rotated for $30 \mathrm{~s}$ at $500 \mathrm{rpm}$ followed by $15 \mathrm{~s}$ at $3000 \mathrm{rpm}$. In the $80 \mathrm{~nm}$ case a resting time of $2 \mathrm{~s}$ was used before

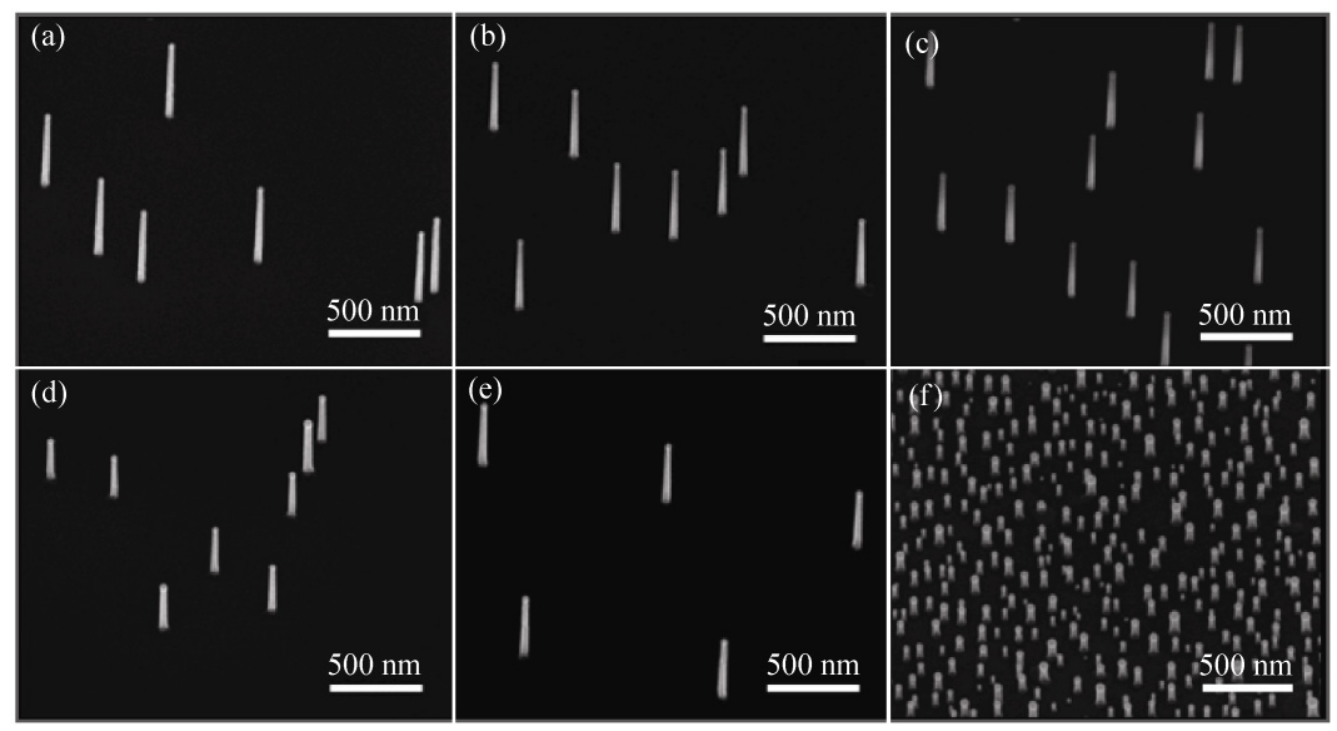

Figure 1 SEM images $\left(30^{\circ}\right.$ tilting angle) of nanowires grown at $430{ }^{\circ} \mathrm{C}$ for 4 min seeded with $30 \mathrm{~nm}$ sized (a) SDA, (b) ECA, (c) DDC, (d) ESC, (e) EBD, and (f) TFA particles 
spinning for $5 \mathrm{~s}$ at $500 \mathrm{rpm}$ followed by $15 \mathrm{~s}$ at $3000 \mathrm{rpm}$. For the electrospray method (TSI, model 3480) the colloidal solutions with a concentration of $4 \times 10^{12}$ and $1.5 \times 10^{12}$ particles $/ \mathrm{cm}^{3}$ for particle diameters of 30 and $80 \mathrm{~nm}$ respectively, were soaked through a capillary and atomized by an electric field into small droplets carrying approximately one particle each. An ultra-pure nitrogen carrier gas was used to dry the particles and transport them to the electrostatic precipitator (ESP; TSI, model 3089) which focused the charged particles directly onto the substrates that were placed on the collector plate [10].

Aerosol particles were generated by two slightly different methods, the evaporation/condensation method [11] and the spark discharge method [12]. In the evaporation/condensation method a solid piece of gold was evaporated in a high temperature furnace at $1800{ }^{\circ} \mathrm{C}$. The as-produced vapor was then transported away by an ultra-pure nitrogen carrier gas, nucleated upon cooling, and finally agglomerate gold particles were formed by condensation and coagulation of primary particles. A similar mechanism was responsible for the formation of agglomerate gold particles by the spark discharge method. The only difference was that the vapor was formed in an aerosol generator (Palas, model GFG 1000) by a spark discharge between two gold electrodes that caused the electrode material to vaporize. For both methods the generated agglomerate particles were further transported into an aerosol nanoparticle system setup $[11,12]$ where they were reshaped in a furnace into compact spherical particles, size-selected by a differential mobility analyzer (DMA) [13] and deposited onto substrates positioned on the collector plate inside an ESP at a deposition voltage of $6 \mathrm{kV}$.

EBD gold particles were prepared by spin-coating the substrates with the resist ZEP 520A7 at $5000 \mathrm{rpm}$ followed by a baking step of $2 \mathrm{~min}$ at $180^{\circ} \mathrm{C}$. Exposure was done using an EBL system (Raith, model 150) with the deposited dose calibrated to form gold disks that transformed during the annealing step inside the MOVPE reactor into particles with a diameter of 30 and $80 \mathrm{~nm}$ respectively. $o$-Xylene was used for $5 \mathrm{~min}$ before rinsing in isopropyl alcohol (IPA) to develop the exposed samples. Following development, 10 and $50 \mathrm{~nm}$ gold films were thermally evaporated at a rate of $0.1 \mathrm{~nm} / \mathrm{s}$ on the 30 and $80 \mathrm{~nm}$ samples respectively. Liftoff was done using Remover 1165 on a hotplate at $60{ }^{\circ} \mathrm{C}$ before rinsing in DIW.

Nanowire growth was performed in a commercial research MOVPE reactor (EpiQuip, model VP502-RP) at $10 \mathrm{kPa}$ using $\mathrm{H}_{2}$ as a carrier gas and a total reactor flow of 6 liters per min. GaAs nanowires were grown at three different temperatures, namely 380,430 , and $480{ }^{\circ} \mathrm{C}$, using trimethylgallium (TMGa) and arsine $\left(\mathrm{AsH}_{3}\right)$ precursors with precursor molar fractions of $9.2 \times 10^{-6}$ and $5.5 \times 10^{-4}$, respectively, resulting in a V/III ratio of 60 . Most runs included an annealing step prior to growth under arsine at $650{ }^{\circ} \mathrm{C}$ for $6 \mathrm{~min}$ to remove oxide and residues and to form particles from the TFA and EBD samples. Growth was also investigated without annealing. Growth times of $0 \mathrm{~s}$ (only annealing), $10 \mathrm{~s}, 30 \mathrm{~s}, 1 \mathrm{~min}, 2 \mathrm{~min}, 4 \mathrm{~min}, 8 \mathrm{~min}$, $12 \mathrm{~min}$, and $24 \mathrm{~min}$ were used to investigate incubation times, growth rate, and nanowire morphology. To provide a direct comparison between the different particle types, all samples containing different particle types were grown in the same runs, while separate runs were performed for the 30 and $80 \mathrm{~nm}$ particles. Care was taken to ensure that the samples were placed in the area on the susceptor where uniform growth is expected (i.e., without a temperature gradient). In addition, the samples were randomly positioned on the susceptor during the different runs and several growth runs were repeated two or three times in order to confirm reproducibility.

Scanning electron microscopy (SEM; FEI, model Nova Nanolab 600) was used to investigate general morphology, diameter, and length of the nanowires. At least 100 wires, from at least 3 different areas, were measured for each sample and analysis software for SEM images, developed in MATLAB, was used for evaluation. A high resolution transmission electron microscope (HRTEM; JEOL, model 3000F) equipped with a field emission gun and operated at $300 \mathrm{kV}$ was used for detailed investigations of nanowire crystal structure. TEM samples were prepared by gently touching the as-grown sample with a lacey carbon $\mathrm{Cu}$ TEM-grid, thus transferring nanowires from the sample to the grid. Each sample investigated by HRTEM was evaluated by examining a minimum of five nanowires or a minimum of ten particles. 


\section{Results and discussion}

\subsection{General seed particle characteristics}

General differences between gold particle generation and deposition techniques, particularly in control of particle properties, are summarized in Table 1. From the perspective of controllability of particle surface density, i.e., the particle number concentration per area, and particle diameter, the TFA particles differ considerably from the other particle types. From the literature it is known that annealing temperature [14] and film thickness [5] can affect the particle distribution to some extent, with higher temperatures and thicker films yielding lower particle number concentrations and larger diameter particles. Regardless of film thickness, annealing time or annealing temperature, however, it is extremely difficult to achieve a low surface density and to independently control particle diameter and surface density. For an annealing temperature of $650{ }^{\circ} \mathrm{C}$ and a film thickness of $1 \mathrm{~nm}$, we observed a particle surface density of above 70 particles $/ \mu \mathrm{m}^{2}$ and a diameter distribution with particle diameters ranging from a few nanometers to above $100 \mathrm{~nm}$. This high surface density of particles, and the very broad diameter distribution, observed for TFA seeded nanowires allowed for only qualitative comparison with nanowires seeded by the other particle types. Although the TFA particles suffer from extremely poor controllability, this method of producing particles also has advantages. It is a fairly simple, reasonably cheap, and very clean method, especially if the film is deposited in situ (something that is not possible for most of the other particle types). TFA particles can be prepared on most types of substrates regardless of size, so long as the substrate material allows for diffusion and agglomeration of gold at temperatures that are not destructive to the substrate.

Colloid particles with well defined diameters and narrow diameter distributions are commercially available and thus do not suffer from the same diameter controllability problems as the TFA particles. On the other hand, if the direct deposition method is used, an inhomogeneous particle surface coverage is often achieved and it is difficult to produce samples with precisely controlled particle densities. Direct deposition usually requires mechanical spinning of the substrate after application of the colloidal suspension, to avoid unreasonably high particle densities. However, this requires that the substrate is not too thin/fragile, too small or too large. If mechanical spinning of the substrate is not possible, drop casting the colloid solution and rinsing in DIW can also reduce the particle density to some extent. Another method to avoid particle density issues can be to use the electrospray setup, which generates samples with a homogenous distribution of a given particle surface density. In

Table 1 Comparison of general particle characteristics between the different particle types

\begin{tabular}{c|c|c|c|c|c|c|c|c}
\hline Particle type & $\begin{array}{c}\text { Diameter } \\
\text { range }\end{array}$ & $\begin{array}{c}\text { Diameter } \\
\text { control }\end{array}$ & $\begin{array}{c}\text { Density } \\
\text { control }\end{array}$ & $\begin{array}{c}\text { Position } \\
\text { control }\end{array}$ & Throughput & Cleanliness & Simplicity & Cost \\
\hline $\begin{array}{c}\text { Aerosol spark } \\
\text { generated (SDA) }\end{array}$ & Limited & Very good & Very good & Very limited & Reasonable & Very clean & Reasonable & High \\
\hline $\begin{array}{c}\text { Aerosol e/c } \\
\text { generated (ECA) }\end{array}$ & Broad & Very good & Very good & Very limited & Reasonable & Very clean & Reasonable & High \\
\hline $\begin{array}{c}\text { Colloid directly } \\
\text { deposited (DDC) }\end{array}$ & Very broad & Very good & Limited & Uncontrolled & Very high & Contaminated & Simple & Low \\
\hline $\begin{array}{c}\text { Colloid } \\
\text { electrospray } \\
\text { deposited (ESC) }\end{array}$ & Very broad & Very good & Very good & Very limited & Low & Contaminated & Reasonable & High \\
\hline $\begin{array}{c}\text { EBL generated } \\
\text { (EBD) }\end{array}$ & $\begin{array}{c}\text { Limited } \\
\text { (small } \\
\text { difficult) }\end{array}$ & Very good & Very good & Very good & Very low & Contaminated & Reasonable & Very high \\
\hline $\begin{array}{c}\text { Thin film } \\
\text { generated (TFA) }\end{array}$ & Broad & Very limited & Very limited & Uncontrolled & Very high & Very clean & Simple & Medium \\
\hline
\end{tabular}


addition, by using the electrospray method, particles can be deposited onto more or less any type of substrate having a diameter of up to a few centimeters. Such a setup is however rather expensive and more complicated to use than the direct deposition method. The main disadvantages of colloid particles, regardless of deposition method, are contamination problems. Organic remnants from the synthesis and stabilization processes, as well as PLL if the direct deposition method is used, contaminate the particle and substrate surfaces. As well, position control is generally not possible for colloid particles deposited by either method.

The preparation method of aerosol particles is extremely clean compared to the colloid preparation method and results in very pure particles. In addition, the particles have a high crystalline quality and tunable monodisperse diameter. The SDA particles are limited to a maximum diameter of around $35 \mathrm{~nm}$, while the upper limit is around $100 \mathrm{~nm}$ for ECA particles, due in both cases to limitations of the setup used. Precisely controlled depositions of particles of a given diameter and surface density at a reasonable throughput is possible for both methods. An additional advantage of the aerosol deposition method, as for the deposition of colloids by electrospray, involves substrate flexibility. Particles can be deposited onto more or less any type of substrate having a diameter up to several centimeters. However, as for the TFA particles and colloid particles, the exact position of the particles on the substrates cannot be controlled. In addition, the cost and complexity of the setup for the aerosol methods can be seen as disadvantageous.

EBD particles are one of the most controllable particle types. Not only can EBD particles be homogenously prepared with a given tunable diameter and surface density, but the exact position on the substrate can also be controlled. A precise limitation of particle diameter and density is difficult to define, but the EBL process becomes increasingly problematic for decreasing particle diameters and increasing surface densities. However EBD particles also suffer from a number of disadvantages. As for the colloid particles, organic residues from the chemical preparation processes might contaminate the substrate surface. Unlike aerosol deposited particles, EBD particles cannot be prepared on substrates that are too thin, brittle, or otherwise unable to withstand a large number of handling and processing steps. As well, the process needs to be modified slightly depending on substrate material used. Since different materials will result in different amounts of back-scattered electrons, and hence different diameters due to the proximity effect, the EBL process must be calibrated and optimized for the substrate currently in use. Moreover the preparation method itself is costly and tedious resulting in a low throughput of samples.

From the discussion above it is clear that a certain particle type could be beneficial in one way but less useful from another point of view, and therefore it is not always obvious which particle type or deposition method should be used. If the resulting nanowires are to be used for fundamental research into growth mechanisms, for example, precise size and position control might not be necessary. On the other hand, if the nanowire application will be in large scale device design, full control over the nanowire diameter, surface position and crystal structure, as well as a complete removal of chemical residues and a reasonable throughput are of major importance. Regardless of application area, it is important to note that different generation and deposition methods yield particles with different size, homogeneity, surface density and cleanliness.

\subsection{Incubation times}

The most striking difference observed between nanowires seeded with different particle types is variations in incubation time, defined as the time from when the growth precursors are turned on until the nanowires are observed to grow. A general trend of shorter incubation times with increasing growth temperature was observed for all particle types of either 30 or $80 \mathrm{~nm}$ diameters. However, significant differences were observed when comparing incubation times between the differently generated and deposited particles. In Table 2, a summary of incubation times is shown, where the incubation step is divided into three parts: "no", "stubs", and "wires". "No" means that no nanowire growth could be observed; the particles seem to be identical to particles on samples that are only annealed. For "stubs" and "wires", growth is 
Table 2 Incubation behavior of the different particle types for growth durations of $10 \mathrm{~s}$ to 4 min at 380,430 and $480{ }^{\circ} \mathrm{C}$ for (a) 30 and (b) $80 \mathrm{~nm}$ sized particles. NO indicates no growth whereas STUBS and WIRES indicate growth below and above approximately $150 \mathrm{~nm}$ respectively. ${ }^{* *}$ The TFA particles have a broad size distribution and much higher particle density, and should not be quantitatively compared to the other particle types

(a)

\begin{tabular}{|c|c|c|c|c|c|c|c|c|c|c|c|c|c|}
\hline \multirow{2}{*}{$\begin{array}{l}\text { Particle } \\
\text { type }\end{array}$} & \multirow{2}{*}{$\begin{array}{c}\text { Growth } \\
\text { temperature }\end{array}$} & \multicolumn{5}{|c|}{ Growth time } & \multirow{2}{*}{$\begin{array}{l}\text { Particle } \\
\text { type }\end{array}$} & \multirow{2}{*}{$\begin{array}{l}\text { Growth } \\
\text { temperature }\end{array}$} & \multicolumn{5}{|c|}{ Growth time } \\
\hline & & $10 \mathrm{~s}$ & $30 \mathrm{~s}$ & $1 \mathrm{~min}$ & $2 \min$ & $4 \min$ & & & $10 \mathrm{~s}$ & $30 \mathrm{~s}$ & $1 \mathrm{~min}$ & $2 \min$ & $4 \mathrm{~min}$ \\
\hline \multirow{3}{*}{ SDA } & 380 & - & - & Stubs & Wires & Wires & \multirow{3}{*}{${ }^{* *}$ TFA } & 380 & - & - & No & No & Stubs \\
\hline & 430 & No & Stubs & Wires & Wires & Wires & & 430 & No & No & No & Stubs & Wires \\
\hline & 480 & Stubs & Wires & Wires & Wires & Wires & & 480 & No & No & Stubs & Stubs & Wires \\
\hline \multirow{3}{*}{ ECA } & 380 & - & - & Stubs & Wires & Wires & \multirow{3}{*}{ ECA } & 380 & - & - & - & - & - \\
\hline & 430 & No & No & Wires & Wires & Wires & & 430 & No & Stubs & Wires & Wires & Wires \\
\hline & 480 & No & Stubs & Wires & Wires & Wires & & 480 & No & Stubs & Wires & Wires & Wires \\
\hline \multirow{3}{*}{$\mathrm{DDC}$} & 380 & - & - & No & Stubs & Wires & \multirow{3}{*}{$\mathrm{DDC}$} & 380 & - & - & - & - & - \\
\hline & 430 & No & No & Stubs & Wires & Wires & & 430 & No & No & No & Stubs & Wires \\
\hline & 480 & No & Stubs & Stubs & Wires & Wires & & 480 & No & No & No & Stubs & Wires \\
\hline \multirow{3}{*}{ ESC } & 380 & - & - & No & Stubs & Wires & \multirow{3}{*}{ ESC } & 380 & - & - & - & - & - \\
\hline & 430 & No & No & Stubs & Wires & Wires & & 430 & No & Stubs & Wires & Wires & Wires \\
\hline & 480 & No & Stubs & Wires & Wires & Wires & & 480 & No & Stubs & Wires & Wires & Wires \\
\hline \multirow{3}{*}{ EBD } & 380 & - & - & No & Stubs & Stubs & \multirow{3}{*}{ EBD } & 380 & - & - & - & - & - \\
\hline & 430 & No & No & Stubs & Stubs & Wires & & 430 & No & No & Stubs & Wires & Wires \\
\hline & 480 & No & No & No & Wires & Wires & & 480 & No & No & No & Wires & Wires \\
\hline
\end{tabular}

observed: growth of less than $150 \mathrm{~nm}$ is designated "stubs" and greater than $150 \mathrm{~nm}$ is denoted "wires". This division between "stubs" and "wires" is an arbitrary definition made here solely in order to make the discussion of variations in incubation time more clear.

SDA particles have the shortest incubation time for all three growth temperatures compared to the other $30 \mathrm{~nm}$ particle types. At $380{ }^{\circ} \mathrm{C}$ small nanowire stubs are observed after $1 \mathrm{~min}$ of growth thus giving an incubation time below $60 \mathrm{~s}$. For the growth temperatures of 430 and $480{ }^{\circ} \mathrm{C}$, the SDA particles have incubation times between 10 and $30 \mathrm{~s}$ and below $10 \mathrm{~s}$, respectively. This is significantly shorter than we observe for the $30 \mathrm{~nm}$ EBD particles, which showed the longest incubation times. For this particle type incubation times between 2 and $4 \mathrm{~min}, 1$ and $2 \mathrm{~min}$, and 1 and $2 \mathrm{~min}$ for the respective growth temperatures are observed. As seen from Table 2, the incubation time increased for the other particle types in the following order: ECA, ESD, and DDC, where ECA and ESD show similar incubation times and DDC particles have incubation times closer to the EBD particles.

Nanowires seeded with $80 \mathrm{~nm}$ sized particles have roughly the same incubation time as the nanowires seeded with $30 \mathrm{~nm}$ sized particles, with some deviations for the colloid particles. Unfortunately, due to limitations of the spark discharge generation method mentioned above, $80 \mathrm{~nm}$ particles of this type (SDA) could not be produced and hence are not compared to the other $80 \mathrm{~nm}$ particle types. In the $80 \mathrm{~nm}$ case, the ECA and ESC particles have the shortest incubation times for all three growth temperatures (see Fig. 2 for the results at $430{ }^{\circ} \mathrm{C}$ ). One would, however, expect the SDA particles to have the shortest incubation time if this could be tested for the $80 \mathrm{~nm}$ particles, following the trend for the $30 \mathrm{~nm}$ particles discussed above. One major difference from the $30 \mathrm{~nm}$ case is that the DDC particles are by far the slowest, for all three growth temperatures, with an even longer incubation time than for the EBD particles.

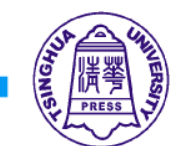

Springer 


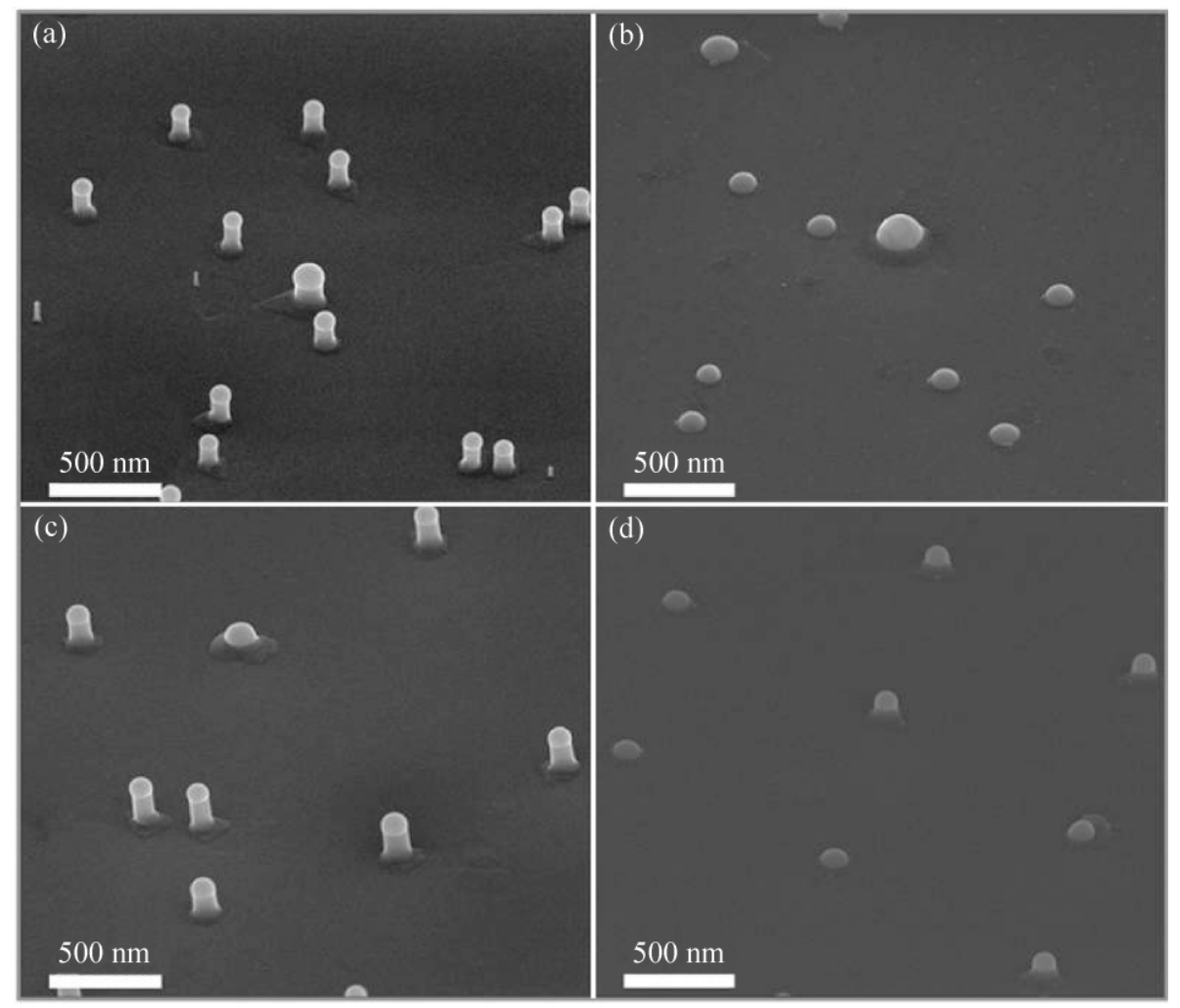

Figure 2 SEM images $\left(52^{\circ}\right.$ tilting angle) of incubation differences at $430{ }^{\circ} \mathrm{C}$ after $1 \mathrm{~min}$ of growth for $80 \mathrm{~nm}$ sized (a) ECA, (b) DDC, (c) ESC, and (d) EBD particles

Incubation times of TFA seed particles are difficult to quantitatively compare to the other particle types, since this technique yields a broad diameter distribution and high particle density. Nevertheless, incubation times between 2 and $4 \mathrm{~min}, 1$ and $2 \mathrm{~min}$ and just above 30 s for the growth temperatures of 380,430 , and $480{ }^{\circ} \mathrm{C}$, respectively, were observed.

It is not surprising that the aerosol generated seed particles have the shortest incubation times since they are very pure and are generated and deposited without involvement of any other chemicals. This is however also true for the TFA seed particles, but due to the very high surface density the individual particles may compete for growth material and therefore supersaturate more slowly than the other particle types deposited with a much lower surface density. Furthermore, the TFA particles have to form during the annealing and/or growth step whereas the aerosols are already formed before deposition. More intriguing and more difficult to understand is the fact that the SDA aerosols always show a shorter incubation time compared to the ECA aerosols. Since the only difference in deposition and generation between these two methods is the formation step of agglomerate particles we believe that something in this process step is responsible for the as-seen incubation differences. Although the morphology of the final reshaped particles is extremely similar when investigated by TEM, the agglomerate particles differ in shape and primary particle size. These observed agglomerate differences result in small differences during reshaping of the agglomerate particles, something that might affect the incubation behaviors [15]. In addition, ECA particles might be exposed to higher amounts of carbon since the evaporation is performed in a graphite tube while a certain amount of oxygen might be present during the generation process of SDA particles. If the particles are surrounded by a carbon shell it is likely that the carbon can interfere with the interaction of the gold with the substrate and precursors and thus increase the incubation time.

In contrast to the aerosol particle generation process, the generation processes of colloidal particles and EBD particles involve chemicals which leave residues 
on the particle surfaces and on the substrate surface. These residues are expected to change the surface properties of both the particles and the substrate, which might influence the surface diffusion of growth species as well as the incorporation of growth species into the particles, and hence result in longer incubation times. Although most of the residues are expected to evaporate during the annealing and/or growth step [9], some residues might remain thus slowing down the incubation process. In addition carbon remnants may still surround the particles. By using the electrospray deposition method instead of the direct deposition method for the colloidal particles, the amount of organic residues surrounding the particles is reduced. In Fig. 3, $80 \mathrm{~nm}$ sized DDC and ESC particles after $10 \mathrm{~s}$ of growth at $480{ }^{\circ} \mathrm{C}$ are displayed; some residues are visible on the DDC particles whereas no residues can be observed on the ESC particles. This could explain why the ESC particles have an incubation time almost as short as the ECA particles. Moreover it is reasonable to believe that this is one of the reasons for the shorter incubation time observed for the electrospray colloids compared to the directly deposited ones. Another factor that should be considered is the PLL layer required for the direct deposition method. Not only does the PLL layer add to the amount of organic residues, but perhaps more importantly it changes the surface properties of the substrate significantly [16]. However, the amount of PLL remains the same for deposition of both 30 and $80 \mathrm{~nm}$ sized colloids and could therefore not explain the prolonged incubation time for the $80 \mathrm{~nm}$ DDC particles. From TEM investigations we found that the particle structure is similar for both particle sizes. We therefore conclude that the most likely explanation for the prolonged incubation time is the larger amount of organic residues observed on the substrate after deposition of the $80 \mathrm{~nm}$ DDC particles.

Similar to the colloid particles, as mentioned above, samples with EBD particles also contain a large amount of organic residues that affect the particle and substrate surfaces, which can be seen as one reason for the longer incubation times. In addition, as for the TFA seeds, particles are formed during the annealing and/or growth step from the gold disks produced by the EBL.

An important particle characteristic that seems to be related to the observed incubation differences may be the carbon content in and/or surrounding the particles, which may interfere with the gold-substrateprecursor interaction, hence increasing the incubation time. As mentioned above nanowires seeded with the purest particle type, namely aerosol generated particles, demonstrate shorter incubation times than nanowires seeded with particle types where carbon-containing chemicals are involved in the preparation. In addition, the longer incubation time for directly deposited compared to electrospray deposited colloids, may be attributed to the PLL layer and the larger amount of residues surrounding the DDC particles, which therefore are probably surrounded by more carbon. Finally, the likeliness of lower carbon incorporation in the SDA compared to the ECA particles during
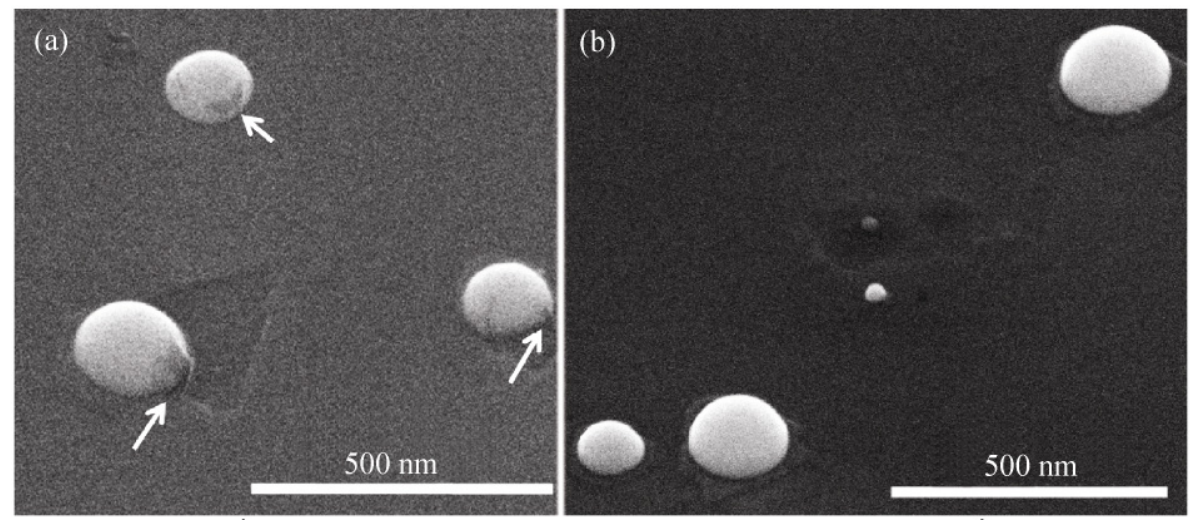

Figure 3 SEM images ( $52^{\circ}$ tilting angle) of $80 \mathrm{~nm}$ sized (a) DDC and (b) ESC particles after annealing at $650{ }^{\circ} \mathrm{C}$ for 6 min followed by $10 \mathrm{~s}$ of growth at $480{ }^{\circ} \mathrm{C}$. White arrows point towards remaining residues 
generation, further strengthens this hypothesis since SDA particle seeded nanowires showed the shortest incubation times of all particle types.

Another particle characteristic that could affect incubation time is the crystal structure of the particles, since defects and grain boundaries may affect diffusion of precursor material through the particle. As mentioned above, no clear difference was observed between the SDA and ECA particles or between the 30 and $80 \mathrm{~nm}$ sized colloids. However, differences were observed between aerosol particles and colloid particles. The aerosols normally contain fewer grain boundaries than the colloids, which typically display a decahedral crystal structure (Fig. 4). It should be noted however, that the crystal structure might change during the annealing and/or growth step. Due to the in situ formation of the TFA and EBD particles inside the MOVPE reactor before growth these could not easily be studied by TEM, and no conclusions could be drawn about the crystal structure effect on nanowire incubation time. In order to provide further insight, more TEM studies would be needed. Moreover in situ TEM studies of the actual nucleation event of nanowires from the different particle types would be extremely interesting and would provide a better understanding of the incubation behavior.

\subsection{Nanowire growth}

The general nanowire growth characteristics including overall growth rate, tapering and side facets of the wires were found to be reasonably similar for nanowires seeded with the various particle types. Regardless of seed particle type the nanowires, which all have $\{112\}$-oriented sidewalls consisting of alternating $\{111\} \mathrm{A}$ and $\{111\} \mathrm{B}$ microfacets, grew straight and perpendicular in the $\langle 111\rangle \mathrm{B}$ direction for most growth conditions used (Fig. 1), with only a few exceptions. One exception concerns all particle types at the growth temperature of $380{ }^{\circ} \mathrm{C}$. At this temperature some of the nanowires seeded with $30 \mathrm{~nm}$ particles kinked during growth and it was impossible to grow freestanding straight nanowires from $80 \mathrm{~nm}$ seed particles. These results are not surprising since $380{ }^{\circ} \mathrm{C}$ is a rather low temperature for growth of $\mathrm{GaAs}$ nanowires [17] and because nanowires with diameters as large as $80 \mathrm{~nm}$ are known to be more sensitive to growth conditions than slightly thinner nanowires. Another exception to successful growth involves growth runs performed without annealing and will be further discussed below.

For all types of seed particles of either 30 or $80 \mathrm{~nm}$ size, the overall nanowire growth rate increased with growth temperature as expected [17] (Fig. S-1 in the Electronic Supplementary Material (ESM) shows the results for $30 \mathrm{~nm}$ sized particles). The overall growth rate is defined as the measured nanowire length divided by the nanowire growth time for the different growth durations - in other words the average growth

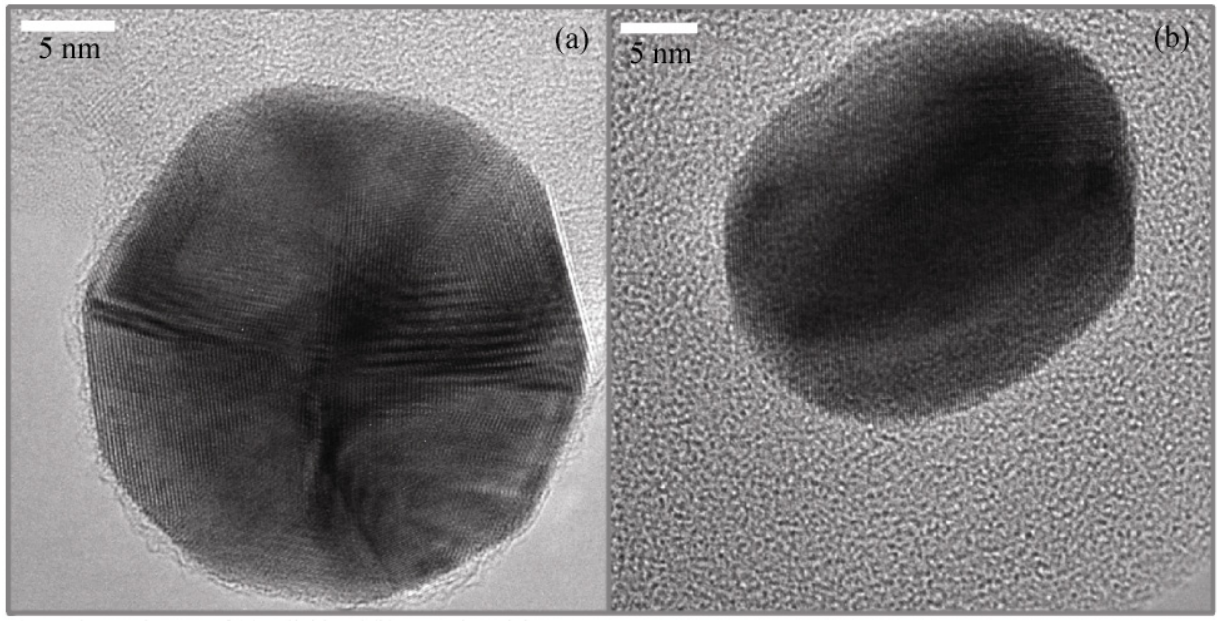

Figure 4 TEM images of (a) colloid and (b) aerosol particles 
rate without accounting for variations with time or length, such as incubation. Despite the differences in incubation time between the various particle types, no clear differences in nanowire length were observed after growth times of $4 \mathrm{~min}$ and longer (with the exception of nanowires seeded with TFA particles) indicating a similar overall nanowire growth rate for all particle types (Fig. S-1 in the ESM). Small deviations are seen for certain data points. These are attributed to occasional not fully perfect particle preparations and irreproducible fluctuations in the system. Although the incubation time variations were clearly visible for short growth durations they were too small (smaller than the error bar of the final length) to have a statistically significant effect on the final nanowire length at longer growth durations. Nanowires seeded with TFA particles were significantly shorter than nanowires seeded with the other particle types, which we attribute to the much higher surface density of this particle type resulting in a lower local supply of growth species. In order to verify the hypothesis that the higher surface density of particles, rather than particle type, is responsible for the shorter nanowires ECA particles were deposited with a similar surface density as the TFA particle samples. As expected, nanowires seeded from these high density samples decorated with ECA particles exhibited a reduced growth rate compared to the ECA samples with a density of 1 particle $/ \mu \mathrm{m}^{2}$.

When investigating the tapering, i.e., non-seeded lateral growth on the nanowire side walls that often results in conically-shaped nanowires, we observed little difference between the nanowires seeded with different particle types. As expected, tapering increases with increasing growth temperature, since layer growth conditions are approached. The only pronounced tapering difference between nanowires seeded with different particle types are observed for the TFA-seeded nanowires. Nanowires seeded with TFA particles as well as the nanowires grown from the high density ECA particle sample exhibit no observable tapering for any of the growth temperatures. We again attribute the observed differences between these two samples and the other samples to the higher surface density of particles.

The major difference between particle types with respect to nanowire growth was found in their behavior during annealing. Annealing was performed under group $\mathrm{V}$ pressure and a rather high annealing temperature of $650{ }^{\circ} \mathrm{C}$ was used in order to achieve TFA particles with the lowest possible surface coverage. This high annealing temperature has no noticeable effect on the $30 \mathrm{~nm}$ sized particles but affected the $80 \mathrm{~nm}$ sized particles to some extent. For the $80 \mathrm{~nm}$ EBD particles, movement of the particles on the substrates was apparent in some cases since wire positions were observed to deviate from the defined pattern (Fig. 5(a)). This is most likely true also for the $80 \mathrm{~nm}$ particles of the other types but due to the random deposition of particles on the substrate no movement could be identified. However, for all $80 \mathrm{~nm}$ particle types under almost all growth conditions a small proportion of the particles split up into larger and smaller particles.

To test the effect of annealing on nanowire growth, several runs were performed without annealing by
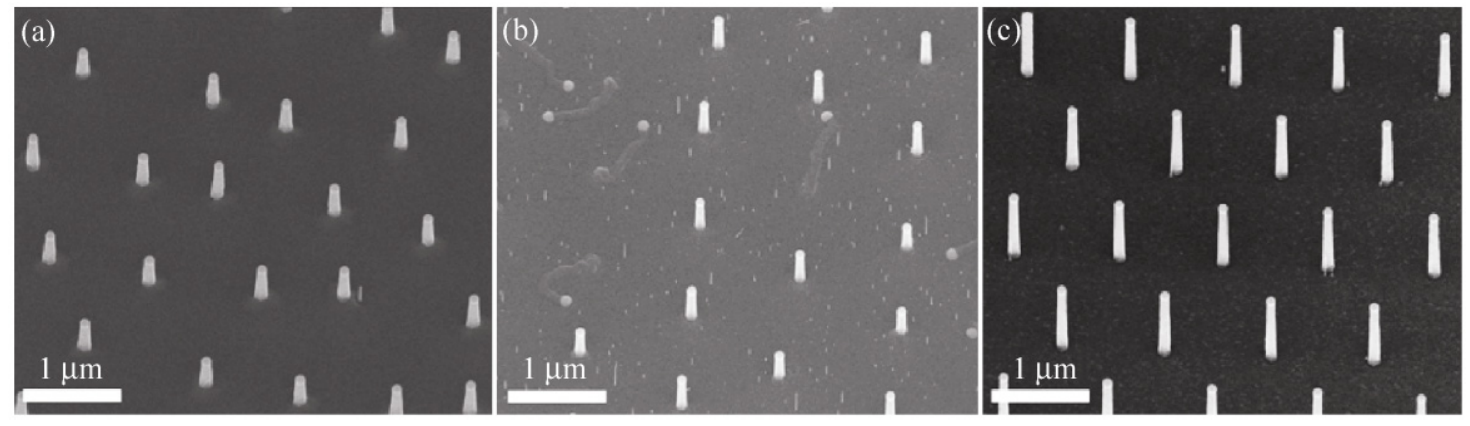

Figure 5 SEM images $\left(30^{\circ}\right.$ tilting angle) displaying annealing effects for $80 \mathrm{~nm}$ sized EBD particles grown for 4 min at (a) $480{ }^{\circ} \mathrm{C}$ with annealing, (b) $430{ }^{\circ} \mathrm{C}$ without annealing, and (c) $480{ }^{\circ} \mathrm{C}$ without annealing 
heating directly to the growth temperature. Nanowires seeded with aerosol and colloidal particles showed successful straight and perpendicular nanowire growth even without the annealing step at all temperatures. Without annealing, however, they were slightly shorter, most likely since evaporation of residues and possible gold-substrate interactions have to occur at the growth temperature instead of at the annealing temperature before the growth. An even larger effect with respect to growth quality and nanowire yield was observed when using EBD and TFA particles. When an initial annealing step was excluded before nanowire growth from the TFA particles, a larger amount of "crawling" and kinked wires was obtained for all growth temperatures compared to the growth runs containing the annealing step. Apparently a certain temperature is needed for appropriate particle formation to take place before the growth precursors can be turned on. A majority of the nanowires seeded with $30 \mathrm{~nm}$ sized EBD particles grown at 380 and $430{ }^{\circ} \mathrm{C}$ were kinked, extremely short, or not nucleated at all. At $480{ }^{\circ} \mathrm{C}$, successful nanowire growth was obtained. For nanowires seeded with $80 \mathrm{~nm}$ sized EBD particles, most wires were "crawling" with only a small number of free-standing straight wires at $430{ }^{\circ} \mathrm{C}$ (Fig. $5(\mathrm{~b})$ ). At $480{ }^{\circ} \mathrm{C}$ on the other hand, even more successful nanowire growth compared to the same conditions with the annealing step was obtained since no movement of particles occurred and the EBD particle pattern remained (Fig. 5(c)). These results indicate that a certain temperature is needed for successful nanowire growth from EBD particles to take place. The particles need to form and residues need to be evaporated, at the same time as particle movement needs to be prevented. However, an "annealing" step as low as $480{ }^{\circ} \mathrm{C}$ may be sufficient for growth of nanowires at lower temperatures with EBD particles.

As mentioned in the introduction of this section, very small differences in general nanowire growth characteristics appear between nanowires seeded with the various particle types. The overall growth rate, microfaceting and tapering behavior are almost the same. The major differences are found in the incubation time and effect of the annealing step, since the different particle generation and deposition methods affect surface properties and hence precursor surface diffusion differently. It is however important to be aware of the number density and size of particles - since both seem to have a larger effect than particle type itself-on characteristics such as overall growth rate and tapering. These results indicate that direct comparison of most nanowire growth characteristics between nanowires seeded with different particle types can be performed, as long as the growth conditions, particle number density and particle size are similar.

\subsection{Nanowire crystal structure}

The crystal structure of nanowires is important if the nanowires are to be used for device applications. In a number of recent reports control of nanowire crystal structure by growth parameter tuning [18], addition of dopant species [19] and seed particle diameter tuning [20] have been demonstrated. From TEM investigations of the crystal structure of nanowires seeded with the different types of particles it was found that seed particle type does not affect nanowire crystal structure. Regardless of particle type (with the exception of nanowires seeded with TFA particles) all nanowires grown at $430^{\circ} \mathrm{C}$ exhibit cubic zinc blende (ZB) structure with a similar density of randomly distributed twin defects (Fig. 6(c) shows the results for ECA particles as an example). The number of twin defects is reduced at the growth temperature of $380{ }^{\circ} \mathrm{C}$ where the nanowires exhibit almost periodic twinning (Fig. 6(b)). On the other hand, at $480{ }^{\circ} \mathrm{C}$ the density of twin defects is enhanced and stacking faults appear resulting in a mixture of $\mathrm{ZB}$ and hexagonal wurtzite (WZ) crystal structures with ZB still clearly predominating (Fig. 6(d)). No pronounced difference between nanowires seeded with 30 and $80 \mathrm{~nm}$ seed particles was found.

Nanowires seeded with TFA particles also exhibit cubic ZB structure but contain a significantly lower density of twin planes compared to nanowires seeded with the other particle types at both 430 and $480{ }^{\circ} \mathrm{C}$. At $380^{\circ} \mathrm{C}$ they are almost completely free of twin planes rather than displaying the regular twinning observed for the nanowires seeded with the other particle types (Fig. 6(a)). However, again this is attributed to the much higher surface density of particles compared to 


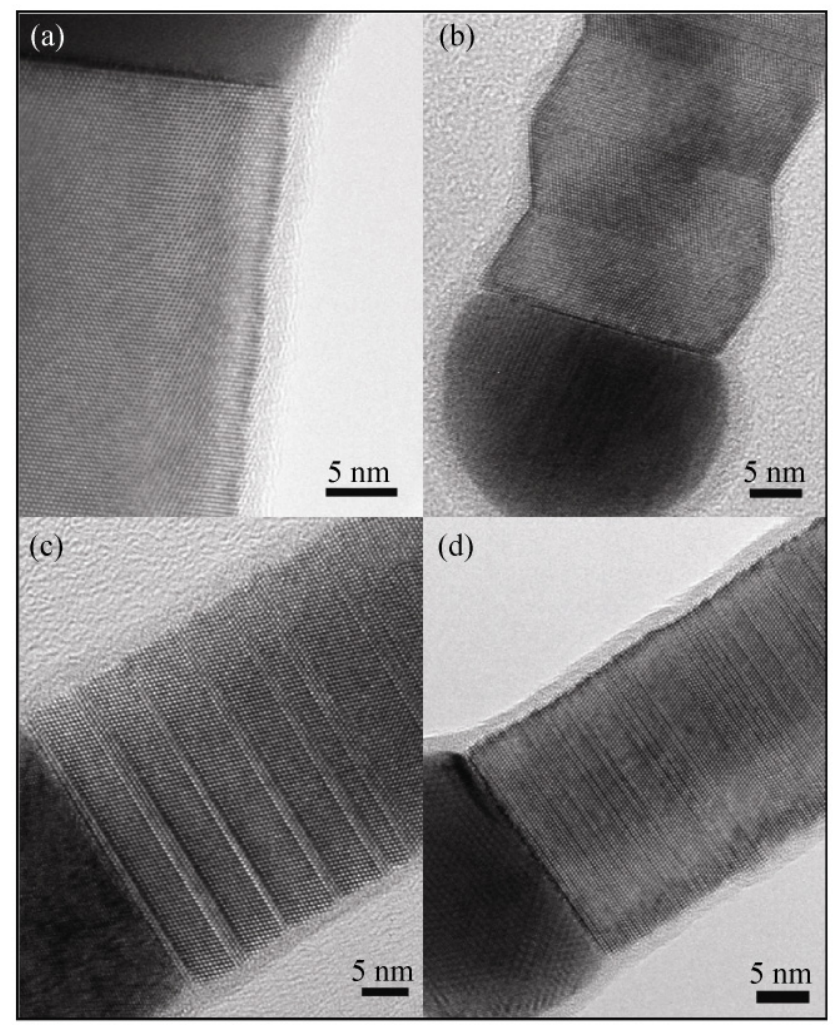

Figure 6 Nanowire crystal structure displayed by TEM. (a) Nanowire grown from TFA particle at $380{ }^{\circ} \mathrm{C}$, (b)-(d) nanowires grown from ECA particles (representative for all particle types apart from TFA particles) at 380,430 and $480{ }^{\circ} \mathrm{C}$ respectively

the other samples resulting in a very different local supersaturation which is known to affect crystal structure [21]. This was confirmed since nanowires seeded from the high density ECA particle sample, with a similar surface coverage to that of the TFA particle samples, exhibited a reduction of twin planes to a level roughly the same as for the TFA particle samples (Fig. 7).

\section{Conclusions}

Nanowire growth induced by gold seed particles is by far the most common method reported for generation of epitaxial semiconductor nanowires. Successful nanowire growth has been reported from a number of different research groups using different types of gold particles, with respect to generation and deposition methods, as seeds. In this article we have presented a direct comparison between nanowires

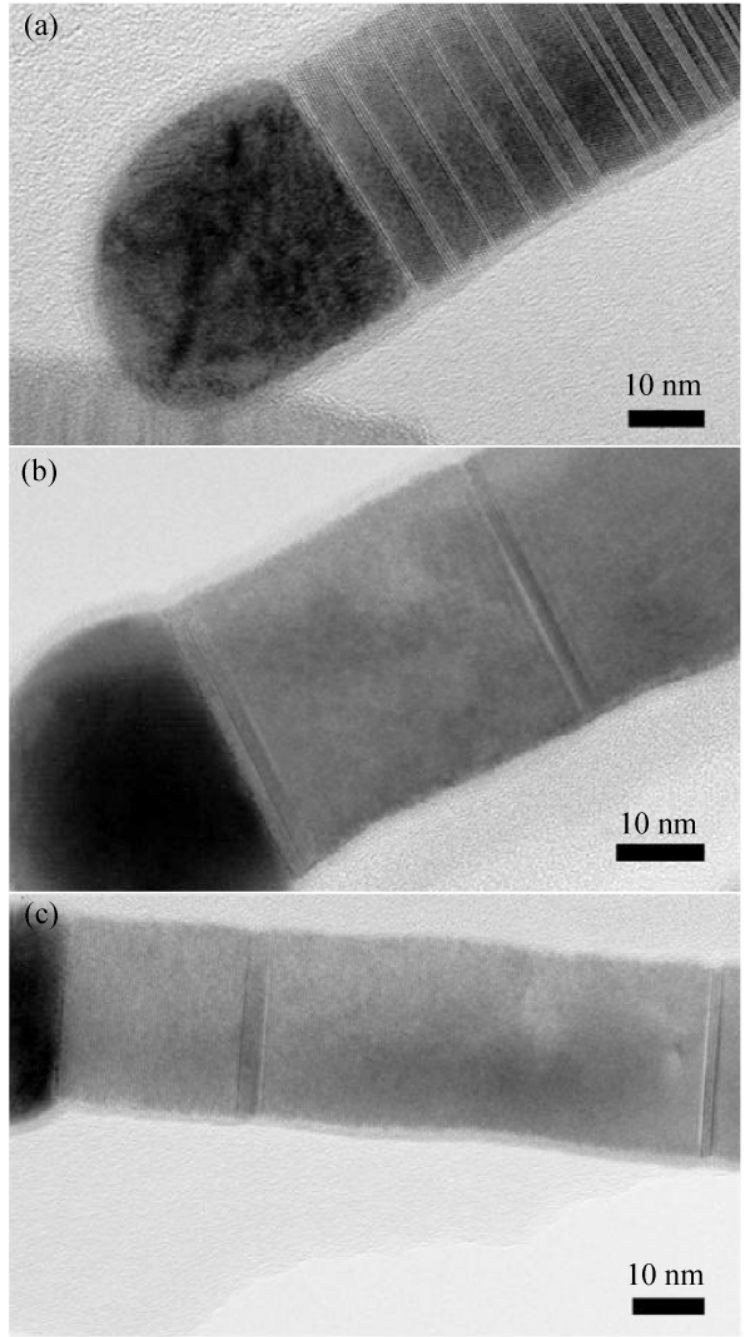

Figure 7 TEM images of nanowire crystal structure for nanowires grown at $430{ }^{\circ} \mathrm{C}$ seeded with (a) ECA particles with density $1 / \mu \mathrm{m}^{2}$ (representative for all other particle types apart from TFA), (b) TFA particles with density $70 / \mu^{2}$, and (c) ECA particles with density $70 / \mu \mathrm{m}^{2}$

seeded with gold particles generated by annealing of thin films, colloidal gold particles deposited by the direct deposition method as well as by the electrospray method, aerosol gold particles generated by evaporation/condensation as well as by spark discharge and EBL-defined gold particles. Nanowires seeded with these different particle types exhibit highly similar growth behavior and structure. The largest differences between particle types are observed in nanowire incubation time and sensitivity to annealing. As long as particle diameter and particle density are comparable, no differences in overall nanowire growth 
rate or crystal structure are observed. This strongly indicates that direct comparisons of nanowire growth studies done in similar systems can be performed, even when different types of gold particles have been used as seeds for the wires. However, it is important to note that the various particle generation and deposition methods themselves suffer from different limitations, which sometimes make it hard to precisely control important characteristics such as density and particle diameter. Furthermore, investigations of optical, electronic and transport properties are needed to determine whether differences in gold seed particle type affect such properties despite the similarities of general growth characteristics and structure observed here.

\section{Acknowledgements}

This work was performed within the Nanometer Structure Consortium at Lund University and supported by the Swedish Research Council (VR), the Swedish Foundation for Strategic Research (SSF), the EU program AMON-RA (No. 214814) and the Knut and Alice Wallenberg Foundation. Furthermore this report is based on a project which was funded by E. ON AG as part of the E. ON International Research Initiative. Responsibility for the content of this publication lies with the authors. The authors acknowledge M. Graczyk for assistance with thin film evaporation and M. T. Borgström and A. Gustafsson for valuable discussions.

Electronic Supplementary Material: Supplementary material including diagrams of the nanowire growth rate is available in the online version of this article at http://dx.doi.org/10.1007/s12274-010-0011-y and is accessible free of charge.

Open Access: This article is distributed under the terms of the Creative Commons Attribution Noncommercial License which permits any noncommercial use, distribution, and reproduction in any medium, provided the original author(s) and source are credited.

\section{References}

[1] Li, Y.; Qian, F.; Xiang, J.; Lieber, C. M. Nanowire electronic and optoelectronic devices. Mater. Today 2006, 9, 18-27.

[2] Messing, M. E.; Hillerich, K.; Johansson, J.; Deppert, K.; Dick, K. A. The use of gold for fabrication of nanowire structures. Gold Bull. 2009, 42, 172-181.

[3] Caroff, P.; Messing, M. E.; Borg, B. M.; Dick, K. A.; Deppert, K.; Wernersson, L. -E. InSb heterostructure nanowires: MOVPE growth under extreme lattice mismatch. Nanotechnology 2009, 20, 495606.

[4] Hochbaum, A. I.; Fan, R.; He, R. R.; Yang P. D. Controlled growth of $\mathrm{Si}$ nanowire arrays for device integration. Nano Lett. 2005, 5, 457-460.

[5] Hiruma, K.; Haraguchi, K.; Masamitsu, Y.; Madokoro, Y.; Katsuyama, T. Nanometre-sized GaAs wires grown by organo-metallic vapour-phase epitaxy. Nanotechnology 2006, 17, S369-S375.

[6] Mårtensson, T.; Borgström, M.; Seifert, W.; Ohlsson, B. J.; Samuelson, L. Fabrication of individually seeded nanowire arrays by vapour-liquid-solid growth. Nanotechnology 2003, 14, 1255-1258.

[7] Mårtensson, T.; Carlberg, P.; Borgström, M.; Montelius, L.; Seifert, W.; Samuelson, L. Nanowire arrays defined by nanoimprint lithography. Nano Lett. 2004, 4, 699-702.

[8] Morales, A. M.; Lieber, C. M. A laser ablation method for the synthesis of crystalline semiconductor nanowires. Science 1998, 279, 208-211.

[9] Böttger, P. H. M.; Bi, Z.; Adolph, D.; Dick, K. A.; Karlsson, L. S.; Karlsson, M. N. A.; Wacaser, B. A.; Deppert, K. Electrospraying of colloidal nanoparticles for seeding of nanostructure growth. Nanotechnology 2007, 18, 105304.

[10] Deppert, K.; Schmidt, F.; Krinke, T.; Dixkens, J.; Fissan, H. Electrostatic precipitator for homogenous deposition of ultrafine particles to create quantum-dot structures. J. Aerosol Sci. 1996, 27, S151-S152.

[11] Magnusson, M. H.; Deppert, K.; Malm, J. -O.; Bovin, J. -O.; Samuelson, L. Gold nanoparticles: Production, reshaping, and thermal charging. J. Nanopart. Res. 1999, 1, 243-251.

[12] Messing, M. E.; Dick, K. A.; Wallenberg, L. R.; Deppert, K. Generation of size-selected gold nanoparticles by spark discharge-for growth of epitaxial nanowires. Gold Bull. 2009, 42, 20-26.

[13] Knutson, E. O.; Whitby, K. T. Aerosol classification by electric mobility: Apparatus, theory and applications. J. Aerosol Sci. 1975, 6, 443-451. 
[14] Plante, M. C.; Garrett, J.; Ghosh, S. C.; Kruse, P.; Schriemer, H.; Hall, T.; LaPierre, R. R. The formation of supported monodisperse $\mathrm{Au}$ nanoparticles by UV/ozone oxidation process. Appl. Surf. Sci. 2006, 253, 2348-2354.

[15] Nakaso, K.; Shimada, M.; Okuyama, K.; Deppert, K. Evaluation of the change in the morphology of gold nanoparticles during sintering. J. Aerosol Sci. 2002, 33, 1061-1074.

[16] Mikkelsen, A.; Eriksson, J.; Lundgren, E.; Andersen, J. N.; Weissenreider, J.; Seifert, W. The influence of lysine on $\mathrm{InP}(001)$ surface ordering and nanowire growth. Nanotechnology 2005, 16, 2354-2359.

[17] Borgström, M.; Deppert, K.; Samuelson, L.; Seifert, W. Size- and shape-controlled GaAs nano-whiskers grown by MOVPE: A growth study. J. Cryst. Growth 2004, 260, 18-22.

[18] Joyce, H. J.; Gao, Q.; Tan, H. H.; Jagadish, C.; Kim, Y.;
Zhang, X.; Guo, Y.; Zou, J. Twin-free uniform epitaxial GaAs nanowires grown by a two-temperature process. Nano Lett. 2007, 7, 921-926.

[19] Algra, R. E.; Verheijen, M. A.; Borgström, M. T.; Feiner, L. -F.; Immink, G.; van Enckevort, W. J. P.; Vlieg, E.; Bakkers, E. P. A. M. Twinning superlattices in indium phosphide nanowires. Nature 2008, 456, 369-372.

[20] Caroff, P.; Dick, K. A.; Johansson, J.; Messing, M. E.; Deppert, K.; Samuelson, L. Controlled polytypic and twinplane superlattices in III-V nanowires. Nat. Nanotechnol. 2009, 4, 50-55.

[21] Johansson, J.; Karlsson, L. S.; Dick, K. A.; Bolinsson, J.; Wacaser, B. A.; Deppert, K.; Samuelson, L. Effects of supersaturation on the crystal structure of gold seeded III-V nanowires. Cryst. Growth Des. 2009, 9, 766-773. 